\title{
The effect of body mass index and fasting glucose on the relationship between blood pressure and incident diabetes mellitus: a 5-year follow-up study
}

\author{
Won Young Lee ${ }^{1,6}$, Chang Hee Kwon ${ }^{2,6}$, Eun Jung Rhee ${ }^{1}$, Jeong Bae Park ${ }^{3}$, Young Kwon Kim ${ }^{4}$, \\ Sook Young $\mathrm{Woo}^{5}$, Seonwoo $\mathrm{Kim}^{5}$ and Ki Chul Sung ${ }^{2}$
}

There is no consensus on the relationship between high blood pressure (BP) and incident diabetes mellitus (DM). Therefore, the aim of the current study was to investigate the independent association between BP and incident DM and identify the metabolic components that influence incident DM in Korean subjects. The current study included 14054 non-diabetic subjects (mean age of 41 years) at the start of the study who were followed for an average of 5 years. We measured the risk for incident DM according to the subjects' baseline BP. Subjects were separated into three groups as follows: normotensive $(<120 /$ $80 \mathrm{~mm} \mathrm{Hg}$ ), pre-hypertensive $(120 / 80 \mathrm{~mm} \mathrm{Hg} \leqslant B P<140 / 90 \mathrm{~mm} \mathrm{Hg}$ ) and hypertensive ( $\geqslant 140 / 90 \mathrm{~mm} \mathrm{Hg}$ ). The overall incidence of DM was $1.8 \%$ (246 subjects), comprising $0.9 \%$ of the normotensive group, $1.9 \%$ of the pre-hypertensive group and $4.0 \%$ of the hypertensive group $(P<0.01)$. Within the hypertensive group, subjects with high body mass index (BMI) and high fasting-glucose levels were 40 times more likely to develop DM compared with those with low BMI and low glucose levels ( 0.3 vs. $13.2 \%, P=0.001$ ). The risk for incident $D M$ was significantly higher in the hypertensive group compared with that in the normotensive group (OR 3.41 vs. 1.00, $P<0.0001$ ). However, the significance disappeared after making adjustments for the baseline BMI and fasting glucose levels (OR 1.18 vs. $1.00, P=0.83$ ). We found that the significance of high $B P$ in predicting incident DM was influenced by the baseline BMI and fasting glucose levels of the subjects.

Hypertension Research (2011) 34, 1093-1097; doi:10.1038/hr.2011.89; published online 14 July 2011

Keywords: body mass index; fasting glucose; high blood pressure; incident diabetes mellitus

\section{INTRODUCTION}

Hypertension often co-exists with type 2 diabetes mellitus (DM). ${ }^{1-4}$ It is estimated that $40-80 \%$ of diabetic patients have a twofold increased risk of hypertension compared with that of normoglycemic individuals. ${ }^{1,3}$ Furthermore, the co-existence of hypertension and DM, such as in patients with metabolic syndrome, increases the risk of cardiovascular disease by approximately twofold over 5-10 years compared with subjects without metabolic syndrome. ${ }^{5}$ A multifactorial treatment approach is essential for the prevention of cardiovascular disease for patients with DM as observed in recent intervention trials. ${ }^{6}$

A few studies have suggested that high blood pressure (BP) is a risk factor for the later development of type $2 \mathrm{DM}^{7,8}$ In the Women's Health Study, baseline BP is an independent predictor for the new-onset of type $2 \mathrm{DM}$ among women that had been healthy at baseline after adjusting for multiple confounding factors such as body mass index (BMI) but they did not include baseline glucose level as a confounder. ${ }^{7}$
The Framingham Offspring Study has reported that a family history of diabetes, obesity, and metabolic syndrome also predicts the development of type $2 \mathrm{DM}$; however, hypertension only had a marginal impact on the development of diabetes after adjusting for glucose and BMI. ${ }^{8}$

Despite the numerous studies that have attempted to clarify the relationship between high $\mathrm{BP}$ and the development of DM, no consensus has been reached concerning whether high BP itself or other metabolic components that affect hypertension and diabetes concurrently influence the development of type $2 \mathrm{DM}$ in Asian subjects with high baseline BP. Furthermore, little is known about the relationship between the relative degree of hypertension and the later development of DM. Therefore, we hypothesized that the risk for the development of type 2 DM was higher in subjects with elevated baseline BP. We investigated the relationship between BP and incident $\mathrm{DM}$ and identified metabolic components that influenced the risk of incident DM in an initial large cohort of healthy Korean subjects.

\footnotetext{
${ }^{1}$ Department of Endocrinology and Metabolism, Kangbuk Samsung Hospital, Sungkyunkwan University School of Medicine, Seoul, Republic of Korea; ${ }^{2}$ Department of Cardiology, Kangbuk Samsung Hospital, Sungkyunkwan University School of Medicine, Seoul, Republic of Korea; ${ }^{3}$ Department of Medicine, Cheil Hospital, Kwandong University School of Medicine, Seoul, Republic of Korea; ${ }^{4}$ Division of Cardiology, Department of Internal Medicine, College of Medicine, Dongguk University, Gyeongju, Republic of Korea and ${ }^{5}$ Department of Biostatistics, Samsung Biomedical Research Institute, Seoul, Republic of Korea

${ }^{6}$ These authors contributed equally to this work.

Correspondence: Dr KC Sung, Division of Cardiology, Department of Internal Medicine, Kangbuk Samsung Hospital, University School of Medicine, 108 Pyeong-dong, Jongno-gu, Seoul 110-746, Republic of Korea.

E-mail: kcmd.sung@samsung.com

Received 8 September 2010; revised 15 March 2011; accepted 10 April 2011; published online 14 July 2011
} 


\section{METHODS}

\section{Subjects}

We retrospectively reviewed the electronic medical records of subjects (mean age of $41.2 \pm 6.1$ years, males were 41.6 \pm 5.9-years old, females were $40.2 \pm 6.5$-years old) who had participated regularly in a general health checkup program that was run through a health promotion center at the Kangbuk Samsung Hospital in Seoul, Korea, between 2003 and 2008. The purposes of the medical health checkup program were to promote the health of employees through regular health checkups and to detect diseases at an early stage. Most of the examinees were employees and their families from various industrial companies across the country. The cost of these medical examinations was largely paid by the employers, and a considerable proportion of the examinees were seen annually or biannually. Therefore, we were able to follow-up with these subjects for 5 years.

\section{Study design}

A total of 15638 subjects were included. Among them, subjects with a known history of diabetes $(N=179)$, a fasting plasma glucose level $\geqslant 6.99 \mathrm{mmoll}^{-1}$ $\left(126 \mathrm{mg} \mathrm{dl}^{-1}\right)$ that was detected during the initial evaluation in $2003(N=382)$, or those with missing medical information $(N=241$ lacked alcohol use history, 361 lacked a smoking record, 309 lacked exercise data and 581 lacked other necessary data) were excluded from the study. Finally, 14054 individuals were selected for the analyses. This study was approved by the Institutional Review Board of the Kangbuk Samsung Hospital.

The health status evaluation consisted of a full medical history, physical examination and a comprehensive blood test. From the available data, we extracted the following information: demographics (age and gender), anthropometric measurements (height and weight), BP (systolic and diastolic) and pulse rate. The heights and weights of the subjects were measured when the subjects were dressed in examination gowns without shoes. The BMI was calculated as the weight in kilograms divided by the squared value of the height in meters $\left(\mathrm{kg} \mathrm{m}^{-2}\right)$. The social history was obtained using a self-questionnaire. Smoking history was categorized into never smoked, currently smoke or smoked in the past. Exercise status was categorized by some exercise (subjects who performed any type of regular exercise at least once a week) and no exercise.

\section{Measurement of laboratory data}

BP was measured using a standardized sphygmomanometer after at least $5 \mathrm{~min}$ of rest according to the Hypertension Detection and Follow-up Program protocol. ${ }^{9}$ Participants were divided into three groups based on their baseline $\mathrm{BP}$ status according to the seventh report of the Joint National Committee on prevention, detection, evaluation and treatment of high BP (JNC 7). These categories included normotensive (systolic $\mathrm{BP}<120 \mathrm{~mm} \mathrm{Hg}$ and diastolic $\mathrm{BP}$ $<80 \mathrm{~mm} \mathrm{Hg}$ ), pre-hypertensive $(120 / 80 \mathrm{~mm} \mathrm{Hg} \leqslant \mathrm{BP}<140 / 90 \mathrm{~mm} \mathrm{Hg})$ and hypertensive $(\geqslant 140 / 90 \mathrm{~mm} \mathrm{Hg}$ or presently taking anti-hypertensive medication) groups. Newly developed DM was defined as a fasting glucose $\geqslant 126 \mathrm{mg} \mathrm{dl}^{-1}$ based on a laboratory examination in 2008 or the self-reported use of anti-diabetic drugs.

Laboratory examinations were performed after at least $12 \mathrm{~h}$ of fasting. Glucose was measured using the hexokinase method (Advia 1800 Autoanalyzer; Bayer Diagnostics, Leverkusen, Germany) with an intra-measurement coefficient of variance of $0.98-1.34 \%$. An enzymatic calorimetric test (Advia 1800, Autoanalyzer; Bayer Diagnostics) was used to measure the total cholesterol and triglyceride concentrations using a coefficient of variance of $1.32-1.55 \%$ for total cholesterol and $0.89-1.38 \%$ for triglycerides. The selective inhibition method was used to measure high-density lipoprotein cholesterol concentration (Advia 1800 Auto-analyzer; Bayer Diagnostics) using a coefficient of variance of $2.64-3.03 \%$.

\section{Statistical analysis}

Descriptive data are presented as the means \pm standard deviation, the median with interquartile range if the parameters were non-parametrically distributed, or the numbers of subjects with percentages within parentheses. The $\chi^{2}$-test was used to compare the proportion of the subjects between different groups divided by the dichotomized groups of metabolic parameters and BP status. One-way analysis of variance and Kruskal-Wallis tests were used to analyze the statistical differences of the mean and median values of various parameters among different groups divided by the BP status.

We used logistic regression to calculate the odds ratios for the development of diabetes after adjusting for confounders. In the multivariate models, we included variables such as baseline age, BMI, plasma glucose level, smoking, alcohol consumption and regular exercise. Interaction of the BP status with baseline BMI and glucose status in the development of diabetes was analyzed using logistic regression analyses with Bonferroni's correction. Receiver-operating characteristics curve analyses were performed to identify the predictors for diabetes. Statistical data analyses were performed using SPSS version 12.0 software (SPSS, Chicago, IL, USA). All of the reported $P$-values were two-tailed, and those less than 0.05 were considered statistically significant.

\section{RESULTS}

At the start of the current study in 2003, $14.9 \%$ of all subjects had hypertension, $39.1 \%$ had prehypertension and $45.9 \%$ had normal BP. Individuals with higher BP tended to be older and had higher BMI, fasting glucose and triglyceride levels and lower high-density lipoprotein cholesterol levels compared with those of subjects with normal BP. A higher percentage of subjects who were male and had an increased alcohol intake and a history of smoking (past or current) were found in the pre-hypertensive and hypertensive groups (Table 1).

During the 5-year follow-up period, 246 (1.8\%) subjects developed DM. The incidences of these new diagnoses occurred at the rates of $4.0 \%$ in the hypertensive group, $1.9 \%$ in the pre-hypertensive group and $0.9 \%$ in the normotensive group (Table 2). The analyses that were performed in the two groups considered baseline BMI and revealed that more subjects in the obese group (BMI $\geqslant 25 \mathrm{~kg} \mathrm{~m}^{-2}$ ) developed diabetes compared with those in the non-obese group (BMI $\left.<25 \mathrm{~kg} \mathrm{~m}^{-2}\right)$, which was independent of BP grouping $(P=0.001)$ (Table 2). Furthermore, new diagnoses of DM frequently occurred within the pre-hypertensive and hypertensive groups compared with those within the normotensive group independent of BMI. There were no interactions between the baseline BMI status and the BP status in the development of DM. In addition, subjects with higher baseline glucose levels were more likely to develop DM independent of BP grouping. Conversely, patients in the hypertensive group developed more diabetes independent of their baseline glucose levels (Table 2). There was a significant interaction between the baseline fasting glucose status and BP status in the development of DM $(P=0.03)$.

BMI and baseline fasting glucose levels were analyzed together in subjects with high baseline BMI and high baseline glucose levels. The results indicate that more subjects in the hypertensive group developed incident DM compared with subjects in the normotensive group ( 13.2 vs. $8.0 \%, P=0.044$ ). When the analyses were conducted only in hypertensive subjects, the subjects with high BMI and high glucose levels were at least 40 times more likely to develop diabetes compared with those with low baseline BMI and low glucose levels (0.3 vs. $13.2 \%, P=0.001$ ) (Table 2 ).

When the risk for the development of DM was analyzed according to the baseline BP status, the hypertensive group had a significantly higher risk of developing diabetes compared with the normotensive group (reference group) with an odds ratio of 3.41 after adjusting for age, sex, alcohol use, smoking status and exercise level (Table 3). However, this significance disappeared when BMI and baseline fasting glucose levels were included as confounders (Table 3). This result is consistent in the analyses of men and women (data not shown).

Receiver-operating characteristics curve analyses were performed using the confounders as predictors for the development of diabetes. The baseline glucose level had the highest area under the receiveroperating characteristics curve value, and BMI had the second highest value (Table 4). 
Table 1 Baseline characteristics of the subjects divided by baseline blood pressure status

\begin{tabular}{|c|c|c|c|c|c|}
\hline & Normotensive group & Pre-hypertensive group & Hypertensive group & Total & P-value ${ }^{a}$ \\
\hline $\begin{array}{l}\text { Number of the participants } \\
(\% ; 95 \% \mathrm{Cl})\end{array}$ & $6457(45.94 ; 45.12-46.77)$ & $5498(39.1 ; 38.31-39.93)$ & 2099 (14.94; 14.35-15.54) & 14054 & - \\
\hline Age (years) & $40.3 \pm 5.6$ & $41.1 \pm 5.8$ & $43.9 \pm 7.3$ & $41.2 \pm 6.1$ & $<0.01$ \\
\hline Sex: male subjects (\%) & 3671 (56.9) & $4452(81.0)$ & $1804(85.9)$ & $9927(70.6)$ & $<0.01$ \\
\hline Fasting blood glucose $\left(\mathrm{mmol} \mathrm{I}^{-1}\right)$ & $5.06 \pm 0.44$ & $5.22 \pm 0.47$ & $5.34 \pm 0.51$ & $5.17 \pm 0.48$ & $<0.01$ \\
\hline Triglyceride $\left(\mathrm{mmol} \mathrm{I}^{-1}\right)$ & $1.15(0.82-1.66)$ & $1.44(1.02-2.03)$ & $1.58(1.15-2.25)$ & $1.32(0.93-1.90)$ & $<0.01$ \\
\hline $\mathrm{BMI}\left(\mathrm{kg} \mathrm{m}^{-2}\right)$ & $22.99 \pm 2.68$ & $24.23 \pm 2.75$ & $25.39 \pm 2.78$ & $23.84 \pm 2.86$ & $<0.01$ \\
\hline HDL-C (mmol I-1) & $1.43 \pm 0.30$ & $1.39 \pm 0.28$ & $1.40 \pm 0.28$ & $1.41 \pm 0.29$ & $<0.01$ \\
\hline SBP (mm Hg) & $104.4 \pm 7.3$ & $119.0 \pm 6.6$ & $134.4 \pm 13.3$ & $114.6 \pm 13.5$ & $<0.01$ \\
\hline DBP (mm Hg) & $67 \pm 5.2$ & $78 \pm 4.0$ & $90 \pm 8.5$ & $74 \pm 10.0$ & $<0.01$ \\
\hline Regular exercise (yes or no) & $2134(33.0)$ & $1979(36.0)$ & $871(41.5)$ & $4984(35.5)$ & $<0.01$ \\
\hline Alcohol (g per day) & $8.2 \pm 12.8$ & $12.1 \pm 15.9$ & $15.5 \pm 18.6$ & $10.8 \pm 15.2$ & $<0.01$ \\
\hline \multicolumn{6}{|l|}{ Smoking history } \\
\hline Never & $3783(58.60)$ & $2454(44.60)$ & 897 (42.70) & $7134(50.80)$ & $<0.01$ \\
\hline Ex & $919(14.20)$ & $1244(22.60)$ & $565(26.90)$ & $2728(19.40)$ & \\
\hline Current & $1755(27.20)$ & $1801(32.80)$ & $637(30.30)$ & $4193(29.80)$ & \\
\hline
\end{tabular}

Abbreviations: BMI, body mass index; DBP, diastolic blood pressure; HDL-C, high-density lipoprotein cholesterol; SBP, systolic blood pressure.

Data are mean $\pm S D$, median (interquartile range) or counts (percentages).

aParameters with parametric distribution are analyzed by one-way ANOVA test, and non-parametrically distributed parameter such as triglyceride level is analyzed by Kruskal-Wallis test. Differences

in proportions of the subjects between the groups are compared with $\chi^{2}$-test.

Table 2 The incidence of newly developed diabetes mellitus according to baseline blood pressure status, body mass index and fasting glucose levels

\begin{tabular}{|c|c|c|c|c|c|}
\hline & Normotensive group (\%) & Pre-hypertensive group (\%) & Hypertensive group (\%) & Total & P-value \\
\hline All subjects & $59 / 6457(0.9)$ & $104 / 5498(1.9)$ & $83 / 2099(4.0)$ & $246 / 14054(1.8)$ & $<0.01$ \\
\hline \multicolumn{6}{|l|}{ Body mass index ${ }^{a}$} \\
\hline$<25 \mathrm{~kg} \mathrm{~m}^{-2}$ & 28/4982 (0.56) & $33 / 3424(0.96)$ & $14 / 945(1.48)$ & 75/9351 $(0.8)^{*}$ & 0.006 \\
\hline$\geqslant 25 \mathrm{~kg} \mathrm{~m}^{-2}$ & $31 / 1475(2.1)$ & $71 / 2074(3.42)$ & $69 / 1154(5.98)$ & $171 / 4703(3.63)^{*}$ & $<0.01$ \\
\hline \multicolumn{6}{|l|}{ Fasting blood glucose ${ }^{\mathrm{b}}$} \\
\hline$\geqslant 5.55 \mathrm{mmol} \mathrm{I}^{-1}$ & $53 / 924(5.7)$ & $91 / 1322(6.9)$ & 73/738 (9.9) & $217 / 2984(7.3)^{*}$ & 0.004 \\
\hline \multicolumn{6}{|l|}{$B M I$ and FBS combined } \\
\hline$<25 \mathrm{~kg} \mathrm{~m}^{-2}$ and $<5.55 \mathrm{mmoll}^{-1}$ & 2/4394 (0.05) & $3 / 736(0.11)$ & 2/670 $(0.3)^{c}$ & 7/7800 (0.09) & 0.114 \\
\hline$\geqslant 25 \mathrm{~kg} \mathrm{~m}^{-2}$ and $\geqslant 5.55 \mathrm{mmoll}^{-1}$ & $27 / 336(8.0)$ & $61 / 634(9.6)$ & $61 / 463(13.2)^{c}$ & 49/1433 (10.4) & 0.044 \\
\hline
\end{tabular}

Abbreviations: BMI, body mass index; FBS, fasting blood sugar.

${ }^{*} P=0.001$ in differences in the proportions between the groups analyzed by $z^{2}$-test.

an interaction analyses, there was no significant interaction between baseline blood pressures status and baseline body mass index status in the development of diabetes.

bIn interaction analyses, there was a significant interaction between baseline blood pressure status and baseline fasting glucose status in the development of diabetes. This significant interaction

was significant even after adjustment for confounding variables such as, age, sex, alcohol, smoking status and exercise status. A total of $5.55 \mathrm{mmol}^{-1}$ is equal to $100 \mathrm{mgdl}-1$.

cWhen $\chi^{2}$-test was performed among the groups with BMI status and FBS status combined and BP status groups, there were significant differences between the groups with $P=0.001$.

\section{DISCUSSION}

In the current study, we found that high baseline BP (pre-hypertension and hypertension) was associated with an increased risk of developing DM over a 5-year follow-up period. However, after adjusting for baseline BMI and fasting glucose levels, this significance disappeared. Therefore, these results suggest that BMI and fasting glucose levels are important factors leading to the development of DM in subjects with hypertension.

Patients with essential hypertension often have impaired glucose tolerance, which is evident in pre-hypertensive subjects. ${ }^{10,11}$ The risk for the development of DM in different groups that were categorized based on baseline BP in the current study was similar to that reported in previous studies. ${ }^{1,7}$ Another recent study that was performed in Turkish subjects has reported the predictability of pre-hypertension for metabolic syndrome, diabetes and coronary artery disease as well as the cardiometabolic risk in women, which is independent of obesity. These findings do not agree with the results of our study. ${ }^{12}$ The main reason for the discrepancy between the previous studies and the present study may be that the previous studies did not adjust for the baseline fasting glucose level, which is one of the most important factors for future incident DM.

The cause for the coincidental occurrence of diabetes and hypertension may be partly explained by the common features of obesity, insulin resistance, increased sympathetic activity and an activated 
Table 3 Odds ratio $(95 \% \mathrm{Cl})$ for the incident DM according to baseline blood pressure status

\begin{tabular}{llcc}
\hline & & & \\
& & Odds ratio (95\% Cl) & P-value \\
\hline \multirow{2}{*}{ Model 1 } & Pre-hypertensive vs. normotensive & $1.77(1.22-2.57)$ & 0.0012 \\
& Hypertensive vs. normotensive & $3.41(2.28-5.10)$ & $<0.0001$ \\
\multirow{2}{*}{ Model 2 } & Pre-hypertensive vs. normotensive & $1.15(0.77-1.72)$ & 0.87 \\
& Hypertensive vs. normotensive & $1.65(1.06-2.56)$ & 0.022 \\
\multirow{2}{*}{ Model 3 3 Pre-hypertensive vs. normotensive } & $1.37(0.94-2.00)$ & 0.127 \\
& Hypertensive vs. normotensive & $1.97(1.30-2.98)$ & 0.0006 \\
\multirow{2}{*}{ Model 5 } & Pre-hypertensive vs. normotensive & $0.97(0.64-1.47)$ & 1.00 \\
& Hypertensive vs. normotensive & $1.18(0.75-1.85)$ & 0.83 \\
\multirow{2}{*}{ Model 6 6 Pre-hypertensive vs. normotensive } & $0.93(0.62-1.40)$ & 1.00 \\
& Pypertensive vs. normotensive & $1.06(0.68-1.66)$ & 1.00 \\
Model 7 & Hypertensive vs. normotensive & $1.08(0.69-1.68)$ & 1.00 \\
& Pre-hypertensive vs. normotensive & $0.96(0.64-1.43)$ & 1.00 \\
& Hypertensive vs. normotensive & $1.10(0.71-1.72)$ & 1.00
\end{tabular}

Abbreviations: $\mathrm{BMI}$, body mass index; $\mathrm{BP}$, blood pressure; $\mathrm{Cl}$, confidence interval; $\mathrm{DM}$, diabetes mellitus.

Model 1: adjust for sex, age, alcohol, smoking and exercise.

Model 2: adjust for model $1+$ fasting glucose.

Model 3: adjust for model 1+ BMI.

Model 4: adjust for model 1+ fasting glucose, BMI.

Model 5: adjust for sex, age, BMI and fasting glucose

Model 6: adjust for sex, BMI and fasting glucose.

Model 7: adjust for BMI and fasting glucose.

Table 4 Area under receiver operating characteristics curves analyses of metabolic parameters predicting future development of diabetes mellitus in all subjects

\begin{tabular}{lccc}
\hline Variables $(\mathrm{n}=14054)$ & AUROC & $95 \% \mathrm{Cl}$ & P-value \\
\hline TG/HDL-C & 0.722 & $0.691-0.754$ & $<0.01$ \\
Fasting glucose & 0.910 & $0.892-0.929$ & $<0.01$ \\
BMI & 0.772 & $0.743-0.801$ & $<0.01$ \\
SBP & 0.710 & $0.680-0.741$ & $<0.01$
\end{tabular}

Abbreviations: AUROC, area under receiver operating characteristics curve; BMI, body mass index; $\mathrm{Cl}$, confidence interval; DM, diabetes mellitus; HDL-C, high-density lipoprotein cholesterol; SBP, systolic blood pressure; TG, triglyceride.

renin-angiotensin-aldosterone system. ${ }^{13,14}$ The causative role of increased sympathetic activity on the development of DM may be supported by a recent study reporting the linear relationship between increased baseline heart rates with the future development of obesity and diabetes in a 20 -year prospective study. ${ }^{15}$ However, this mechanism is challenged by the increased risk for new-onset diabetes in clinical trials using beta-blockers compared with that in clinical trials with other anti-hypertensives. ${ }^{16}$ In contrast, the reduced incidence of new-onset diabetes in trials using angiotensin-converting enzyme inhibitors or angiotensin receptor blockers suggests the deleterious effects of an activated renin-angiotensin system on the development of diabetes. ${ }^{16}$

Racial differences in the characteristics of type 2 DM have been reported. Caucasians had the highest BMI and fat percentage of the four ethnic groups (blacks, whites, Asians and Puerto Ricans). African-Americans had a lower body fat percentage compared with Caucasians. In addition, Asians had lower values compared with Caucasians at a similar age, sex and BMI. ${ }^{17,18}$ Asian people have a tendency to develop DM with a lesser degree of obesity at a younger age and display a 'metabolically obese' phenotype (that is normal body weight with increased abdominal adiposity). ${ }^{19} \mathrm{~A}$ defect in the insulin secretory capacity has been reported more frequently in Asians compared with people of other ethnicities despite similarities in BMI. ${ }^{17,20}$ Among Asians with genetically determined lower levels of insulin secretion, a small increase in weight may accelerate the development of diabetes. In the current study, the increased risk for the development of DM among hypertensive subjects may be attributed to elevated BMI and baseline hyperglycemia. We confirmed that BMI is an important factor to predict the development of type $2 \mathrm{DM}$ in Korean subjects.

The current study had several limitations. First, new-onset DM was defined using the fasting glucose level or a self-reported medical history of anti-diabetic drugs. However, no oral glucose tolerance test was performed. The difference between the previous studies and the present study may be explained by the fact that the present study defined new-onset DM based on fasting glucose levels alone but not post-prandial glucose levels. Baseline hypertension may potentially be used as an independent predictor for incident DM in Asians if newonset DM is defined using fasting glucose and post-prandial glucose levels. Second, although we obtained the medical history for the presence of hypertension at the beginning of the study, we did not determine which medications were being used to treat baseline hypertension in the hypertensive group. Third, a family history of diabetes was not included in the analyses. Fourth, our study cohort was relatively homogenous and predominantly male, consisting of industrial employees or their family members. Therefore, the external validity of our results cannot be determined. In addition, we were more easily able to follow-up with healthy subjects compared with subjects with chronic or serious illnesses, which may have introduced a selection bias into our analyses. Further studies are required to measure the biochemical parameters such as the role of pro-inflammatory cytokines, endothelial function and indices of insulin function in order to identify pathophysiological explanations for our findings. Such studies may help to clarify the metabolic abnormalities that link hypertension and the development of DM.

The current study has several strengths. Our sample size is very large and secondary compared with the sample size used in the Women's Health Study to evaluate the relationship between baseline BP and the development of DM. ${ }^{7}$ Our study is the largest study performed with Asians, and we followed the subjects for a long period. These strengths outweigh any possible biases of our study.

In conclusion, it is important to emphasize intensive lifestyle modifications to prevent new-onset DM in hypertensive patients, especially in patients with high BMI and high glucose levels. Among patients with the same BP, the baseline BMI and glucose levels significantly influence the risk of incident DM. The correction of metabolic components as a whole rather than focusing on BP control alone is a better strategy to prevent the development of DM in hypertensive subjects.

\section{CONFLICT OF INTEREST}

The authors declare no conflict of interest.

\section{ACKNOWLEDGEMENTS}

We acknowledge the efforts of the health-screening group at the Kangbuk Samsung Hospital in Seoul, Korea. This work was partially supported by Samsung Biomedical Research Institute Grant SBRI C-A8-216-1.

1 Lonati C, Morganti A, Comarella L, Mancia G, Zanchetti A. Prevalence of type 2 diabetes among patients with hypertension under the care of 30 Italian clinics of hypertension: results of the (Iper)tensione and (dia)bete study. J Hypertens 2008; 26 : 1801-1808 
2 Hypertension in Diabetes Study (HDS): I. Prevalence of hypertension in newly presenting type 2 diabetic patients and the association with risk factors for cardiovascular and diabetic complications. J Hypertens 1993; 11: 309-317.

3 Tarnow L, Rossing P, Gall MA, Nielsen FS, Parving HH. Prevalence of arterial hypertension in diabetic patients before and after the JNC-V. Diabetes Care 1994; 17: 1247-1251.

4 Hirose H, Saito I. Trends in blood pressure control in hypertensive patients with diabetes mellitus in Japan. Hypertens Res 2003; 26: 717-722.

5 Alberti KG, Eckel RH, Grundy SM, Zimmet PZ, Cleeman JI, Donato KA, Fruchart JC, James WP, Loria CM, Smith Jr SC, International Diabetes Federation Task Force on Epidemiology and Prevention; Hational Heart Lung, and Blood Institute; American Heart Association; World Heart Federation; International Atherosclerosis Society; International Association for the Study of Obesity. Harmonizing the metabolic syndrome: a joint interim statement of the International Diabetes Federation Task Force on Epidemiology and Prevention; National Heart, Lung, and Blood Institute; American Heart Association; World Heart Federation; International Atherosclerosis Society; and International Association for the Study of Obesity. Circulation 2009; 120: 1640-1645

6 Gaede $\mathrm{P}$, Lund-Andersen $\mathrm{H}$, Parving $\mathrm{HH}$, Pedersen $\mathrm{O}$. Effect of a multifactorial intervention on mortality in type 2 diabetes. N Engl J Med 2008; 358: 580-591.

7 Conen D, Ridker PM, Mora S, Buring JE, Glynn RJ. Blood pressure and risk of developing type 2 diabetes mellitus: the Women's Health Study. Eur Heart J 2007; 28: 2937-2943.

8 Wilson PW, Meigs JB, Sullivan L, Fox CS, Nathan DM, D'Agostino RBSr. Prediction of incident diabetes mellitus in middle-aged adults: the Framingham Offspring Study. Arch Intern Med 2007; 167: 1068-1074.

9 Curb JD, Ford C, Hawkins CM, Smith EO, Zimbaldi N, Carter B, Cooper C. A coordinating center in a clinical trial: the Hypertension Detection and Followup Program. Control Clin Trials 1983; 4: 171-186.

10 McFarlane SI, Banerii M, Sowers JR. Insulin resistance and cardiovascular disease. $J$ Clin Endocrinol Metab 2001; 86: 713-718.

$11 \mathrm{Hwu}$ CM, Liou TL, Hsiao LC, Lin MW. Prehypertension is associated with insulin resistance. QJM 2009; 102: 705-711.
12 Onat A, Yazici M, Can G, Kaya Z, Bulur S, Hergenc G. Predictive value of prehypertension for metabolic syndrome, diabetes, and coronary heart disease among Turks. Am J Hypertens 2008; 21: 890-895.

13 Mancia G. The association of hypertension and diabetes: prevalence, cardiovascular risk and protection by blood pressure reduction. Acta Diabetol 2005; 42(Suppl 1): S17-S25.

14 Takeda Y. Pleiotropic actions of aldosterone and the effects of eplerenone, a selective mineralocorticoid receptor antagonist. Hypertens Res 2004; 27: 781-789.

15 Shigetoh $Y$, Adachi $H$, Yamagishi S, Enomoto M, Fukami A, Otsuka M, Kumagae S, Furuki K, Nanjo Y, Imaizumi T. Higher heart rate may predispose to obesity and diabetes mellitus: 20-year prospective study in a general population. Am J Hypertens 2009; 22: 151-155.

16 Alderman $\mathrm{MH}$. New onset diabetes during antihypertensive therapy. Am J Hypertens 2008; 21: 493-499.

17 Ramachandran A, Ma RC, Snehalatha C. Diabetes in Asia. Lancet 2010; 375: 408-418.

18 Wang J, Thornton JC, Burastero S, Shen J, Tanenbaum S, Heymsfield SB, Pierson Jr RN. Comparisons for body mass index and body fat percent among Puerto Ricans, blacks, whites and Asians living in the New York City area. Obes Res 1996; 4 : 377-384.

19 Nishina M, Kikuchi T, Yamazaki H, Kameda K, Hiura M, Uchiyama M. Relationship among systolic blood pressure, serum insulin and leptin, and visceral fat accumulation in obese children. Hypertens Res 2003; 26: 281-288.

20 Itoh K, Imai K, Masuda T, Abe S, Tanaka M, Koga R, Itoh H, Nakamura M. Association between blood pressure and insulin resistance in obese females during weight loss and weight rebound phenomenon. Hypertens Res 2001; 24: 481-487.

This work is licensed under the Creative Commons Attribution-NonCommercial-No Derivative Works 3.0 Unported License. To view a copy of this license, visit http:// creativecommons.org/licenses/by-nc-nd/3.0 